\title{
Initial results of SpineCor treatment of Adolescent Idiopathic Scoliosis in Seville, Spain
}

\section{A Vera Miller}

Address: Centro de Enfermedades de la Columna Vertebral. C/Jáuregui 4-6, portal D, B-2. 41003 Seville, Spain

Email: A Vera Miller - alfvera@telefonica.net

from 6th International Conference on Conservative Management of Spinal Deformities

Lyon, France. 2I-23 May 2009

Published: I4 December 2009

Scoliosis 2009, 4(Suppl 2):O5I doi:I0.II86/I748-7|6I-4-S2-O5 I

This abstract is available from: http://www.scoliosisjournal.com/content/4/S2/O5 I

(c) 2009 Miller; licensee BioMed Central Ltd.

\section{Objective}

The objective of this study was to determine the effectiveness of the SpineCor brace in patients with adolescent idiopathic scoliosis treated in Seville, Spain.

\section{Background}

The SpineCor brace is used at St. Justine Children's Hospital in Montréal. Their results claim that SpineCor is an effective treatment for AIS. We chose to study the effect of this brace on our patients and see if it was similar to the Montreal results.

\section{Methods}

Thirty seven patients were treated using the SpineCor Brace in Seville. Of the 37, 33 patients met criteria of the SpineCorporation international multicenter study treatment protocol. These patients were still under treatment and had not yet achieved a definitive outcome (two years follow-up post brace treatment). The girls were premenarchal or less than 1 year postmenarchal. Effectiveness was looked at using the following parameters: (1) a percentage of patients with an initial Cobb angle reduction of 5 degrees or greater; (2) percentage of patients with an initial Cobb angle increase or decrease of less than 5 degrees; (3) percentage of patients with an initial Cobb increase of 5 degrees or greater; (4) the number of cases progressing to require surgery or undergone surgery.

\section{Results}

At the end of the first year, successful treatment (correction > 5 degrees, or stabilization +/- 5 degrees) was achieved in 32 of the 33 patients studied from the time of fitting of the SpineCor Brace to the point at which that last Cobb angle was measured during bracing. This meant an overall correction and stabilization for $97 \%$ of the patients in Seville, Spain during their first year of treatment. 1 out of 33 patients (3\%) had curve progression of more than 5 degrees and underwent surgery.

\section{Conclusion}

The SpineCor Brace is a potentially effective treatment for adolescent idiopathic scoliosis. We need to continue our study over a longer period until patients achieve a definitive result. However, these initial results seem promising and are similar to the initial results originally achieved at St. Justine Children's Hospital. 